\title{
Levels of the Odd Mass Isotopes of Sb and I and the Unified Model
}

\author{
I. V. Goldstein and A. G. De Pinho \\ Department of Physics, Universidade Católica, Rio de Janeiro, Brasil
}

(Z. Naturforsch. 26 a, 1987-1991 [1971]; received 2 June 1971)

\begin{abstract}
A description of the low-lying positive parity levels of the odd mass isotopes of $\mathrm{Sb}$ and I is presented on the basis of the intermediate coupling approach of the unified model. Such analysis helps to understand many properties of those levels since a rather satisfactory agreement with experimental data is reached.
\end{abstract}

\section{Introduction}

A survey ${ }^{1-10}$ of the low-energy level spectra of the odd mass isotopes of antimony and iodine reveals certain striking features common to the two. Considering the mass ranges, $113 \leqq A \leqq 129$ for antimony and $125 \leqq A \leqq 133$ for iodine, the following interesting aspects become clear:

a) In the lightest isotopes of both elements, the ground state is $5 / 2^{+}$, generally followed by an excited $7 / 2^{+}$state, the relative position of these states being inverted in the heaviest isotopes.

b) The states, $3 / 2(l=2)$ and $1 / 2(l=0)$, are regularly observed with energies not exceeding $1 \mathrm{MeV}$ or so. These levels are characterized by moderate values of the spectroscopic factors in $\left(\mathrm{He}^{3}, \mathrm{~d}\right)$ reactions on the doubly even isotopes of Sn and Te.

c) A group of high spin, ( $I \geqq 7 / 2)$, positive parity levels is seen in many of those nuclides with energies ranging from 600 to about $1600 \mathrm{keV}$. Some of these levels are strongly excited by the inelastic scattering of charged particles.

d) Many other regular features can be noted, related to $\log (f t)$ values for beta feeding, reduced transition probailities, branching ratios, static moments, etc.

Reprint requests to Dr. A. G. DE Pinho, Physics Department, Pontificia Universidade Católica, Rua Marques de Sao Vicente, 209/263, Rio de Janeiro ZC-20, Brasilien.

1 P. D. Barnes, C. Ellegaard, B. Hesskind, and M. C., Joski, Phys. Lett. 23, 266 [1966].

2 M. Conjeaud, S. Harar, and Y. Cassagnou, Nucl. Phys. A117, 449 [1968].

3 G. Berzins, W. H. Kelly, G. Graeffe, and W. B. Walters, Nucl. Phys. A104, 241 [1967].

4 G. Berzins and W. H. Kelly, Nucl. Phys. A104, 263 [1967].

5 P. A. Baedecker and W. B. Walters, Nucl. Phys. A 107, 449 [1968].
It is shown in this paper that at least a qualitative understanding of these several features can be achieved within the framework of a very simple model: the intermediate coupling version of the unified model introduced by BoHR and MottelSON 11,12 , and developed by CHOUdHURY ${ }^{13}$.

\section{Description of the Model}

In the restricted version that will be used here, the intermediate coupling model views the odd mass nuclear system as consisting of an even spherical core plus the unpaired particle. The core is treated as an harmonic vibrator and only quadrupolar phonons will be considered. The Hamiltonian is then written as the sum of a single particle spherical shell model Hamiltonian with eigen energies $\varepsilon(n l j)$, a vibrational Hamiltonian, and a coupling term.

In the approximation of small quadrupolar oscillations about the spherical shape the collective Hamiltonian in the number representation is given by

$$
H=\hbar \omega \sum_{\mu}\left(b_{\mu}^{+} b_{\mu}+1 / 2\right)
$$

where $b_{\mu}^{+}$and $b_{\mu}$ are the usual creation and destruction operators for quadrupolar phonons in the magnetic sub-state $\mu$, and $\hbar \omega$ is the energy for

6 R. L. Auble, J. B. Ball, and C. B. Fulmer, Phys. Rev. 169, 955 [1968].

7 B. Parsa, G. E. Gordon, and W. B. Walters, Nucl. Phys. A110, 674 [1968].

8 A. G. De Pinho, J. M. F. Jeronymo, and I. D. GoldMan, Nucl. Phys. A 116, 408 [1968].

9 M. L. Rustgi and J. G. Lucas, Nucl. Phys. A117, 321 [1968].

10 V. Lopac, Nucl. Phys. A 138, 19 [1968].

11 A. BoHr, Dan. Mat. Fys. Medd. 26, n. ${ }^{\circ} 14$ [1952].

12 A. Bohr and B. Mottelson, Dan. Mat. Fys. Medd. 27, n. 16 [1953].

13 D. C. Choudhury, Dan. Mat. Fys. Medd. 28, n. ${ }^{\circ} 4$ [1954]. 
excitation of a single phonon. If $N$ is the total number of quadrupolar phonons then the energy eigenvalues are

$$
E=\hbar \omega(N+5 / 2) .
$$

The core angular momentum is defined as $R$.

In the same representation the interaction part of the Hamiltonian is given by

$$
H_{\mathrm{int}}=-\xi \hbar \omega(\pi / 5)^{1 / 2} \cdot \sum_{\mu}\left(b_{\mu}+(-1)^{\mu} b_{-\mu}^{+}\right) Y_{2 \mu}(\theta, \Phi)
$$

where $\xi$, the coupling strength, is given in terms of the radial average of the $r$-dependent part of the interaction.

A basis was chosen which diagonalizes the partial Hamiltonian $H^{\prime}=H_{\text {s.p. }}+H_{\text {coll }}$ $|j, N R ; I M\rangle$

$$
=\sum_{\mathrm{m}}\langle j m R M-m \mid I m\rangle|j m\rangle|N R M-m\rangle
$$

and the eigenvectors of the total Hamiltonian were expressed as linear combinations

$$
|I(v) M\rangle=\sum_{j N R} a_{j N R}^{I(v)}|j, N R ; I M\rangle
$$

corresponding to the eigenenergies $E(I, v)$. The ordinal $v$ is assigned from the lowest to the highest, that is, if $v<v^{\prime}$ then $E(I, v)<E\left(I, v^{\prime}\right)$.

The diagonalization space was truncated by considering only the $2 \mathrm{~d} 5 / 2, \lg 7 / 2,2 \mathrm{~d} 3 / 2$ and $3 \mathrm{~s} 1 / 2$ shell model states and three quadrupolar phonons states.

Once the eigenvectors are obtained, the static and dynamic electromagnetic moments are easily calculated.

The model parameters are the energy differences $E_{j}=\varepsilon(n l j)-\varepsilon(2 \mathrm{~d} 5 / 2)$ for the given sets of quantum numbers, $n l j$, the vibrational energies, $\hbar \omega$, and the coupling strength. They were fixed with an energy best fit criterion and the restriction of a smooth variation from one isotope to the next.

Figs. 1 and 2 show how the parameters vary with the neutron number for $Z=51$ and $Z=53$.

\section{Results}

The calculated energy levels are compared with experimental levels in Table 1 . The overall agreement is quite good.

An attempt was made ${ }^{14}$ to apply the model to heavier nuclei in the region $55 \leqq Z \leqq 61$ but, even

14 I. V. Goldstein, M. Sc. Thesis, Universidade Católica, Rio de Janeiro 1970, (unpublished).

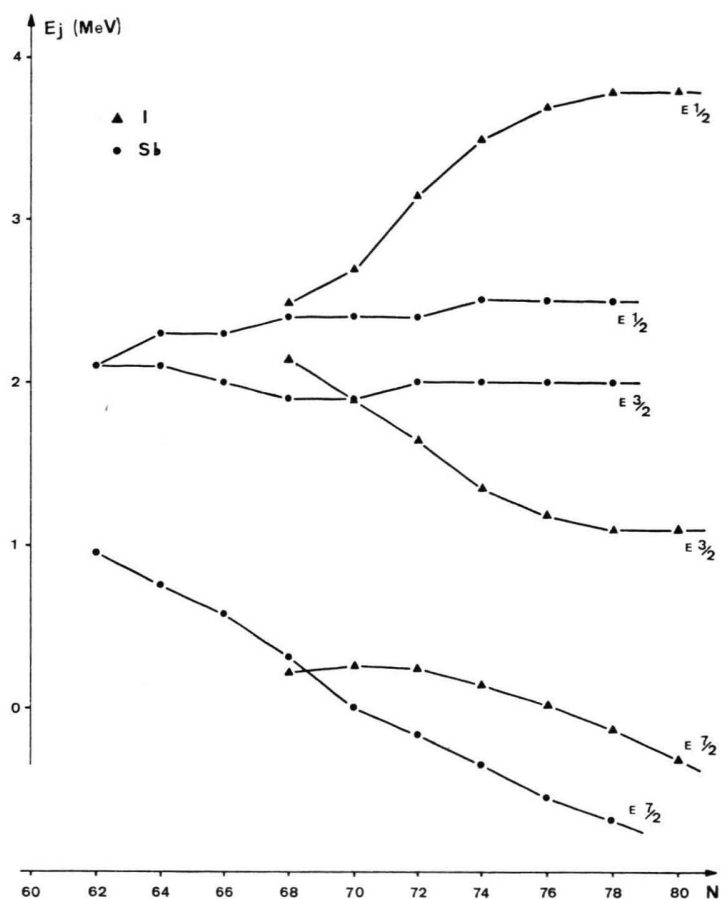

Fig. 1. Variation of the energ y differences $E_{j}$ with the neutron number.

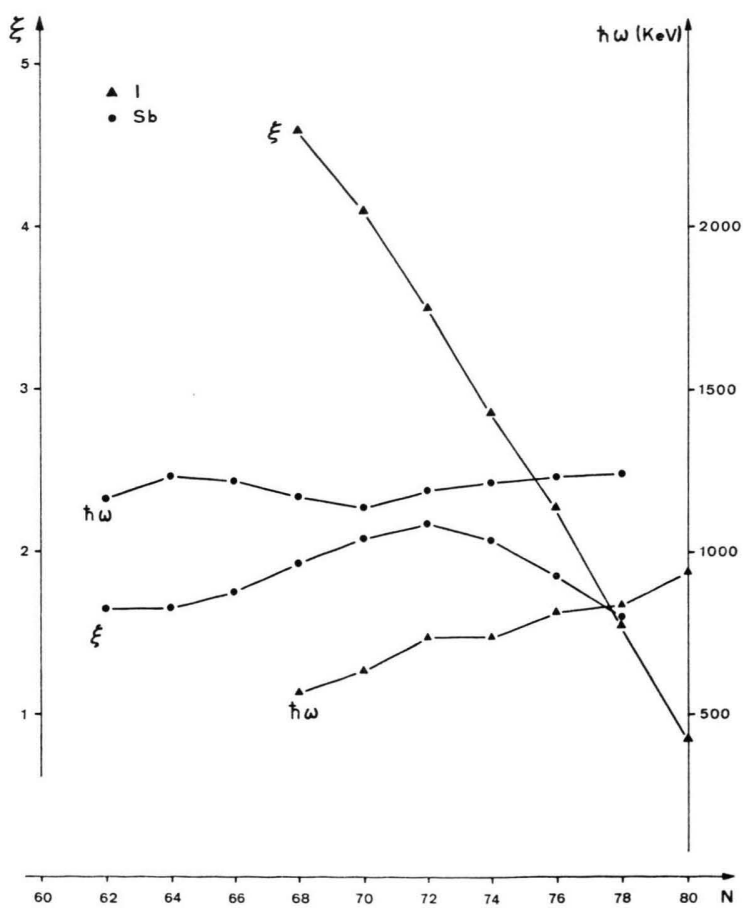

Fig. 2. Variation of the vibrational energies, $\hbar \omega$, and the coupling strength, $\varepsilon$, with the neutron number. 
Table 1. Experimental and calculated positive levels in the odd-A isotopes of Sb and I*.

\begin{tabular}{|c|c|c|c|c|c|}
\hline & $\mathrm{b}^{113}$ & & & $\mathrm{Sb}^{115}$ & \\
\hline Expt. ${ }^{2}$ & Theor & & Expt. 2,17 & Theor. & \\
\hline $0 l=2$ & 0 & $5 / 2(1)$ & $0 l=2$ & 0 & $5 / 2(1)$ \\
\hline $650 l=0$ & 718 & $1 / 2(1)$ & $680 l=4$ & 685 & $7 / 2(1)$ \\
\hline $830 l=4$ & 840 & $7 / 2(1)$ & $780 l=0$ & 771 & $1 / 2(1)$ \\
\hline $1050 l=2$ & 1050 & $3 / 2(1)$ & $1090 l=2$ & 1040 & $3 / 2(1)$ \\
\hline & 1064 & $9 / 2(1)$ & & 1130 & $9 / 2(1)$ \\
\hline & 1188 & $7 / 2(2)$ & & 1220 & $7 / 2(2)$ \\
\hline & 1280 & $5 / 2(2)$ & & 1355 & $5 / 2(2)$ \\
\hline $1590 l=2$ & 1566 & $3 / 2(2)$ & $1380 \quad 3 / 2$ & 1555 & $3 / 2(2)$ \\
\hline & 1851 & $5 / 2(3)$ & & 17851 & $11 / 2(1)$ \\
\hline & 1882 & $11 / 2(1)$ & & 1863 & $9 / 2(2)$ \\
\hline & 1923 & $3 / 2(3)$ & & 1953 & $5 / 2(3)$ \\
\hline & 1953 & $9 / 2(2)$ & & 2019 & $3 / 2(3)$ \\
\hline & $b^{117}$ & & & $\mathrm{Sb}^{119}$ & \\
\hline Expt. ${ }^{2,4}, 14$ & Theor & & Expt. $4,18,19$ & Theor. & \\
\hline $0 l=2$ & 0 & $5 / 2(1)$ & $\begin{array}{ll}0 & 5 / 2\end{array}$ & 0 & $5 / 2(1)$ \\
\hline $520 l=4$ & 521 & $7 / 2(1)$ & $271 \quad 7 / 2$ & 272 & $7 / 2(1)$ \\
\hline $720 l=0$ & 721 & $1 / 2(1)$ & 644 & 644 & $1 / 2(1)$ \\
\hline $924 l=2$ & 918 & $3 / 2(1)$ & 700 & 702 & $3 / 2(1)$ \\
\hline $1160(9 / 2)$ & 1110 & $9 / 2(1)$ & $1048 \quad 7 / 2$ & 1127 & $7 / 2(2)$ \\
\hline & 1196 & $7 / 2(2)$ & $1213 \quad 9 / 2$ & 1046 & $9 / 2(1)$ \\
\hline $1355(l=2)$ & 1358 & $5 / 2(2)$ & $1250 \quad 9 / 2$ & 1386 & $9 / 2(2)$ \\
\hline $1455(l=2)$ & 1500 & $3 / 2(2)$ & & 1332 & $5 / 2(2)$ \\
\hline & 1605 & $11 / 2(1)$ & $1339(1 / 2,3 / 2$ & 1412 & $3 / 2(2)$ \\
\hline & 1700 & $9 / 2(2)$ & $\begin{array}{l}1407(7 / 2, \\
9 / 2,11 / 2)\end{array}$ & 13001 & $11 / 2(1)$ \\
\hline & 1860 & $5 / 2(3)$ & $1413(1 / 2,3 / 2$ & 1412 & $3 / 2(2)$ \\
\hline & 1870 & $7 / 2(3)$ & $1487(3 / 2)$ & 1590 & $7 / 2(3)$ \\
\hline $1716(1 / 2,3 / 2)$ & 1954 & $5 / 2(4)$ & $-\quad-$ & 1616 & $5 / 2(3)$ \\
\hline $1811(1 / 2,3 / 2)$ & 1962 & $3 / 2(3)$ & $1750 \quad(3 / 2)$ & - & - \\
\hline & $b^{121}$ & & & $\mathrm{Sb}^{123}$ & \\
\hline Expt. 1,20 & Theor & & Expt. $1,21,22$ & Theor. & \\
\hline & 0 & $5 / 2(1)$ & $\begin{array}{ll}0 & 7 / 2\end{array}$ & 0 & $7 / 2(1)$ \\
\hline $38 \quad 7 / 2$ & 34 & $7 / 2(1)$ & $1615 / 2$ & 162 & $5 / 2(1)$ \\
\hline $510 \quad 3 / 2$ & 514 & $3 / 2(1)$ & $542 \quad 3 / 2$ & 544 & $3 / 2(1)$ \\
\hline $575 \quad 1 / 2$ & 574 & $1 / 2(1)$ & $712 \quad 1 / 2$ & 701 & $1 / 2(1)$ \\
\hline $9487 / 2,9 / 2$ & 1027 & $11 / 2(1)$ & $\begin{array}{l}1032(7 / 2, \\
9 / 2,11 / 2)\end{array}$ & 1033 & $11 / 2(1)$ \\
\hline $10247 / 2,9 / 2$ & 1114 & $9 / 2(2)$ & $10899 / 2,11 / 2$ & 1110 & $9 / 2(1)$ \\
\hline $10387 / 2,9 / 2$ & 1002 & $9 / 2(1)$ & 1187 & 1210 & $9 / 2(2)$ \\
\hline $11417 / 2,9 / 2$ & 1076 & $7 / 2(2)$ & 1258 & 1247 & $7 / 2(2)$ \\
\hline 1382 & 1320 & $5 / 2(2)$ & & 1416 & $7 / 2(3)$ \\
\hline & 1346 & $7 / 2(3)$ & $1510 l=2$ & 1437 & $5 / 2(2)$ \\
\hline & 1355 & $3 / 2(2)$ & 1574 & 1555 & $5 / 2(3)$ \\
\hline 1446 & & & & 1556 & $3 / 2(2)$ \\
\hline $1623 l=0$ & 1648 & $1 / 2(2)$ & & 1743 & $7 / 2(4)$ \\
\hline & $b^{127}$ & & & $\mathrm{Sb}^{129}$ & \\
\hline Expt. 24 & Theor. & & Expt. ${ }^{24}$ & Theor. & \\
\hline $0 l=4$ & 0 & $7 / 2(1)$ & $0 l=4$ & 0 & $7 / 2(1)$ \\
\hline $498 l=2$ & 500 & $5 / 2(1)$ & $640 l=2$ & 640 & $5 / 2(1)$ \\
\hline $776 l=2$ & 780 & $3 / 2(1)$ & $910 l=2$ & 905 & $3 / 2(1)$ \\
\hline & 1111 & $11 / 2(1)$ & & 11501 & $11 / 2(1)$ \\
\hline & 1160 & $9 / 2(1)$ & & 1180 & $9 / 2(1)$ \\
\hline $1180 l=0$ & 1190 & $1 / 2(1)$ & & 1325 & $7 / 2(2)$ \\
\hline & 1340 & $7 / 2(2)$ & & 1415 & $5 / 2(2)$ \\
\hline & 1444 & $5 / 2(2)$ & $1450 l=0$ & 1446 & $1 / 2(1)$ \\
\hline & 1614 & $9 / 2(2)$ & & 1788 & $9 / 2(2)$ \\
\hline & 1688 & $7 / 2(3)$ & & 1837 & $7 / 2(3)$ \\
\hline & 1850 & $3 / 2(2)$ & & 1959 & $3 / 2(2)$ \\
\hline & 1880 & $5 / 2(3)$ & & 1990 & $5 / 2(3)$ \\
\hline
\end{tabular}

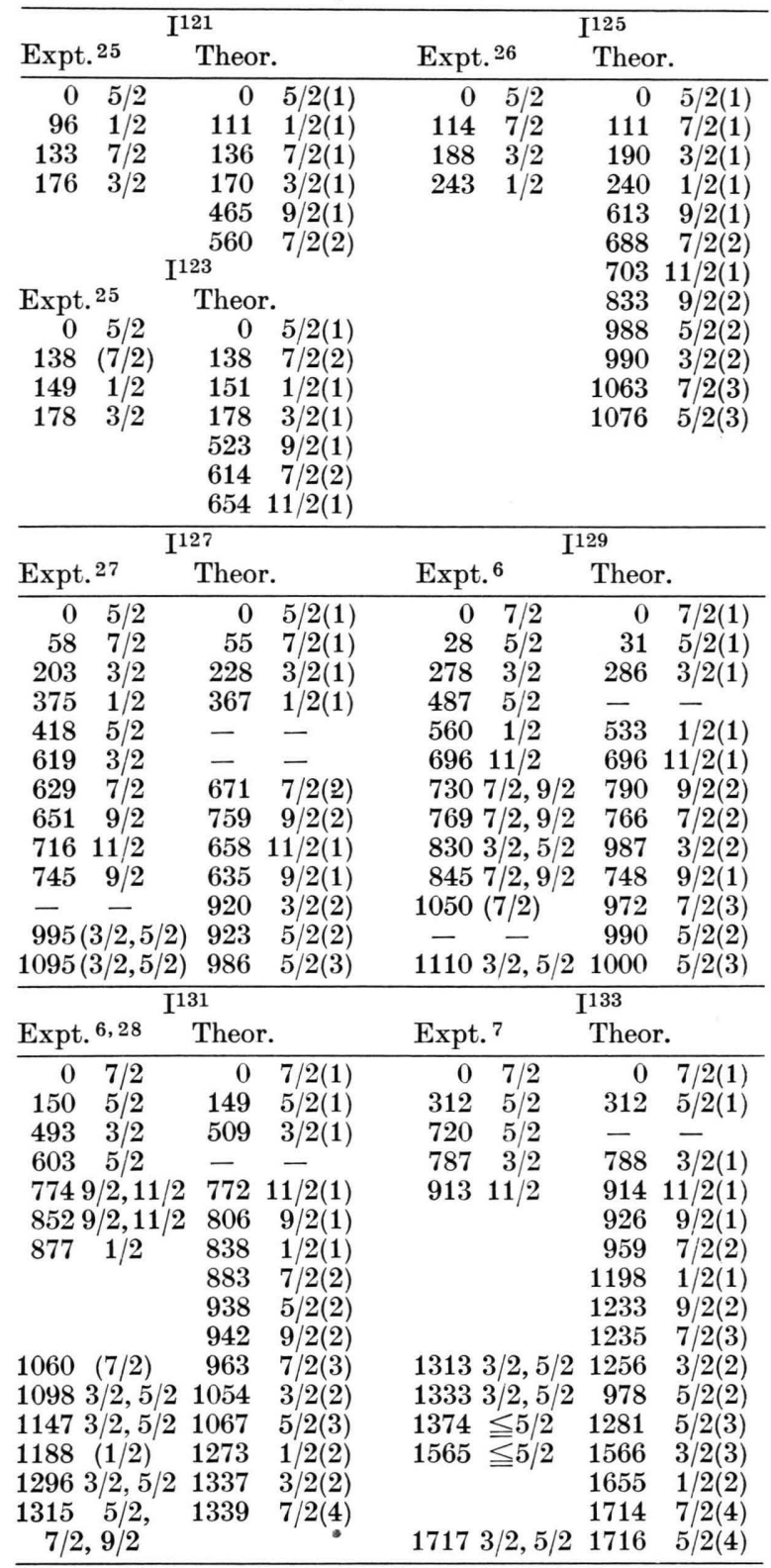

17 R. Reising and B. D. Pate, Nucl. Phys. 61, 529 [1965].

18 T. Ishimatsu, T. Nakagawa, K. Yagi, H. Ohmura, T. Nakajima, and H. Orihara, Nucl. Phys. A104, 481 [1967].

19 G. Graeffe, Phys. Rev. 158, 1183 [1967].

20 R. L. Auble, W. H. Kelly, and H. H. Bolotin, Nucl. Phys. 58, 337 [1964].

21 J. M. F. Jeronymo, N. L. da Costa, A. G. de Pinho I. D. Goldman, and Guillaumon, Nuovo Cim. $55 \mathbf{B}$, 491 [1968].

\footnotetext{
* The first and second columns give, for each nuclide, the observed values of the energies (in keV) and the attributed values of angular momenta, respectively. The third and fourth columns give the calculated values of $E(\mathrm{keV})$ and $I(v)$ for levels which were identified with the experimental ones.
} 
if many qualitative aspects of the energy spectra are well reproduced, we cannot claim a good agreement. It seems that the intermediate coupling model fails if the nucleus presents a very soft core.

Another interesting point, already noted by CHoudhury ${ }^{15}$, is that some low-lying levels appear in iodine which are not present in antimony. The most conspicous of them is the second $5 / 2$ level observed in $\mathrm{I}^{127}, \mathrm{I}^{129}, \mathrm{I}^{131}$ and $\mathrm{I}^{133}$ at $418,487,603$ and $720 \mathrm{keV}$ respectively. They are supposed to correspond to a partial excitation of the core due to the recoupling of the odd particle with a pair of protons moving in the same orbital as the unpaired proton. The mutual interaction of identical particles in the same orbital is sufficiently strong to invalidate, at least partially, the arbitrary separation of the nucleus in an even core containing all the particles but one plus this odd particle.

A detailed discussion of the results will be presented only for $\mathrm{Sb}^{121}$ and $\mathrm{Sb}^{125}$. A more complete collection of results can be found elsewhere ${ }^{16}$.

The squared amplitude of the dominant configurations that appear in the wave functions of the low-lying levels of $\mathrm{Sb}^{125}$ are given in Table 2 to help in understanding some assignments that were made. For instance the observed levels at 1067 and $1087 \mathrm{keV}$ are identified with the calculated levels
$11 / 2(1)$ and $9 / 2(1)$ at 1066 and $1133 \mathrm{keV}$, respectively; they decay preferentially to the ground state. On the other hand a $9 / 2$ level at $1419 \mathrm{keV}$ is identified with the calculated $9 / 2(2)$ state since it decays with a greater probability to the first excited state. Levels at 1484,1700 and $1736 \mathrm{keV}$ are fed by the beta decay of $\operatorname{Sn}^{125}\left(3 / 2^{+}\right)$. We suggest the following assignments: $5 / 2(2), 3 / 2(2)$ and $5 / 2(3)$ respectively, since the first one is observed to decay preferentially to the ground state and the last two levels feed with greater probabilities the first $5 / 2$ state. Such decays are to be interpreted as a core de-excitation and must be associated with enhanced E 2 transitions.

Calculated static and dynamic electromagnetic moments and spectroscopic factors are presented in Table 3 and compared with experimental data for $\mathrm{Sb}^{121}$.

\section{Conclusions}

The intermediate coupling approach to the unified model was used to describe the low-lying $(E \leqq 1.5 \mathrm{MeV})$ positive parity levels of the odd-A isotopes of $\mathrm{Sb}$ and $\mathrm{I}$.

Most of the observed states are explained with considerable success by using very few variable

Table 2. Low-lying positive parity levels of $\mathrm{Sb}^{125}$.

\begin{tabular}{|c|c|c|c|c|c|c|c|}
\hline \multicolumn{2}{|c|}{$\begin{array}{l}\text { Observed level } 5,6,23 \\
\text { Energy }(\mathrm{keV}) \quad I^{\pi}\end{array}$} & \multicolumn{2}{|c|}{$\begin{array}{l}\text { Calculated level } \\
\text { Energy }(\mathrm{keV}) \quad I^{\pi}\end{array}$} & \multicolumn{4}{|c|}{$\begin{array}{l}\text { Squared amplitude of the dominant } \\
\text { configurations }|N R j\rangle \text { in the wave function }\end{array}$} \\
\hline 0 & $7 / 2^{+}$ & 0 & $7 / 2^{+}(1)$ & $|00 \quad 7 / 2\rangle$ & $(74.8 \%)$ & $|12 \quad 7 / 2\rangle$ & $(17.3 \%)$ \\
\hline 332 & $5 / 2^{+}$ & 334 & $5 / 2^{+}(1)$ & $|005 / 2\rangle$ & $(71.9 \%)$ & $|125 / 2\rangle$ & $(15.9 \%)$ \\
\hline 643 & $3 / 2^{+}$ & 644 & $3 / 2^{+}(1)$ & $|127 / 2\rangle$ & $(53.7 \%)$ & $\left|\begin{array}{ll}0 & 3 / 2\end{array}\right\rangle$ & $(17.5 \%)$ \\
\hline 921 & $1 / 2^{+}$ & 925 & $1 / 2^{+}(1)$ & $|125 / 2\rangle$ & $(50.1 \%)$ & $\left|\begin{array}{ll}00 & 1 / 2\end{array}\right\rangle$ & $(15.0 \%)$ \\
\hline 1067 & $(9 / 2,11 / 2)^{+}$ & 1066 & $11 / 2^{+}(1)$ & $\mid \begin{array}{ll}12 & 7 / 2\rangle\end{array}$ & $(72.8 \%)$ & $|24 \quad 7 / 2\rangle$ & $(12.5 \%)$ \\
\hline 1089 & $(11 / 2,9 / 2)^{+}$ & 1133 & $9 / 2^{+}(1)$ & $|12 \quad 7 / 2\rangle$ & $(70.7 \%)$ & $|247 / 2\rangle$ & $(15.7 \%)$ \\
\hline 1349 & $(7 / 2)^{+}$ & 1524 & $7 / 2^{+}(3)$ & $|125 / 2\rangle$ & $(53 \%)$ & $|245 / 2\rangle$ & $(12 \%)$ \\
\hline 1419 & $\left(9 / 2^{+}\right.$ & 1408 & $9 / 2^{+}(2)$ & $|125 / 2\rangle$ & $(70 \%)$ & $|245 / 2\rangle$ & $(14 \%)$ \\
\hline 1484 & $(5 / 2,3 / 2)^{+}$ & 1455 & $5 / 2^{+}(2)$ & $|12 \quad 7 / 2\rangle$ & $(75 \%)$ & $|247 / 2\rangle$ & $(9 \%)$ \\
\hline 1700 & $(1 / 2,3 / 2,5 / 2)^{+}$ & 1708 & $3 / 2^{+}(2)$ & $|125 / 2\rangle$ & $(63 \%)$ & $|247 / 2\rangle$ & $(16 \%)$ \\
\hline 1736 & $(3 / 2,5 / 2)^{+}$ & 1728 & $5 / 2^{+}(3)$ & $|125 / 2\rangle$ & $(50 \%)$ & $|245 / 2\rangle$ & $(8 \%)$ \\
\hline
\end{tabular}

15 D. C. Choudhury and T. F. O'Dwyer, Nucl. Phys. A 93, 300 [1967].

16 A. G. DE Pinho, I. V. Goldstein, and J. M. F. JeroNymo, An. Acad. Bras. Ciênc. (to be published).

22 R. L. Auble and W. H. Kelly, Nucl. Phys. 81, 442 [1966].

23 J. F. Wilds and W. B. Walters, Nucl. Phys. A103, 601 [1967].
24 R. L. Auble, J. B. Ball, and R. B. Fulmer, Nucl. Phys. A 116, 14 [1968].

25 H. Schrader, R. Stippler, and F. Münnich., Nucl. Phys. A 151, 331 [1970].

26 J. S. Geiger, Phys. Rev. 158, 1094 [1967].

27 K. E. Apt, W. B. Walters, and G. E. Gordon, Nucl. Phys. A 152, 344 [1970].

28 L. M. Beyer and W. H. Kelly, Nucl. Phys. A 104, 274 [1967]. 
Table 3. Some properties of the low-lying levels of Sb121. Magnetic moments were calculated with $g_{\mathrm{s}}=0.6 g_{\mathrm{s}}$ (free)and electric moments with $e_{\mathrm{p}}=e$ and $k=33 \mathrm{MeV}$. The spectroscopic factors refer to ( $\mathrm{He}^{3}$, d) reaction on $\mathrm{Sn}^{120}$.

\begin{tabular}{|c|c|c|c|c|}
\hline & Levels & $\begin{array}{l}\text { Calculated } \\
\text { values }\end{array}$ & Experimental values & Ref. \\
\hline \multirow[t]{2}{*}{ Magnetic dipole moment, (n.m.) } & $5 / 2^{(1)}$ & 3.367 & $3.3600 \pm 0.0015$ & 29 \\
\hline & $7 / 2^{(1)}$ & 2.507 & $2.35 \pm 0.03$ & 30 \\
\hline \multirow[t]{2}{*}{ Electric quadrupole moment. $\left(e \times 10^{-24} \mathrm{~cm}^{2}\right)$} & $5 / 2(1)$ & -0.514 & \pm 0.08 & 31 \\
\hline & $7 / 2^{(1)}$ & -0.640 & -0.75 & 31,32 \\
\hline$B$ (M 1), (n.m.) ${ }^{2}$ & $3 / 2^{(1)} \rightarrow 5 / 2^{(1)}$ & 0.32 & $0.13-1$ & 20,33 \\
\hline \multirow[t]{6}{*}{$B(\mathrm{E} 2),\left(e^{2} \times 10^{-48} \mathrm{~cm}^{4}\right)$} & $5 / 2^{(1)} \rightarrow 9 / 2^{(1)}$ & 0.119 & $0.100 \pm 0.016$ & 1 \\
\hline & $5 / 2^{(1)} \rightarrow 7 / 2^{(2)}$ & 0.105 & $0.120 \pm 0.016$ & 1 \\
\hline & $5 / 2^{(1)} \rightarrow 5 / 2^{(2)}$ & 0.029 & \pm 0.005 & 1 \\
\hline & $5 / 2^{(1)} \rightarrow 1 / 2^{(1)}$ & 0.023 & $0.027 \pm 0.003$ & 1 \\
\hline & $3 / 2^{(1)} \rightarrow 7 / 2^{(1)}$ & 0.054 & $0.086 \pm 0.010$ & 34 \\
\hline & $5 / 2^{(1)} \rightarrow 3 / 2^{(1)}$ & 0.010 & $0.007 \pm 0.002$ & 1 \\
\hline \multirow[t]{4}{*}{ Spectroscopic factors } & $5 / 2^{(1)}$ & 0.74 & 0.72 & 2 \\
\hline & $7 / 2^{(1)}$ & 0.73 & 0.70 & 2 \\
\hline & $3 / 2^{(1)}$ & 0.22 & 0.27 & 2 \\
\hline & $1 / 2^{(1)}$ & 0.15 & 0.30 & 2 \\
\hline
\end{tabular}

parameters. The N-dependence of these parameters was not examined in detail; only a microscopic model could account for it. However we observe that a smooth variation of these parameters is a necessary and sufficient condition to reach a rather good overall agreement with experimental data.

29 J. Eisinger and G. Feher, Phys. Rev. 109, 1172 [1958]. 30 S. L. Ruby, G. M. Kalvins, G. B. Beard, and C. E. SNyder, Phys. Rev. 159, 239 [1967].

31 F. Les, Acta Phys. Polon. 20, 775 [1961].

32 S. L. Ruby, G. M. Kalvins, R. E. SNyder, and G. B. Beard, Phys. Rev. 148, 176 [1966].
All the calculations were performed on the IBM-7040 computer of the PUC/RJ.

We would like to thank Prof. J. M. F. Jeronymo for many useful discussions.

This work was supported by grants from CNPq. and BNDE.

33 F. R. Metzger and H. Langhoff, Phys. Rev. 132, 1753 [1963].

34 C. M. Lederer, J. M. Hollander, and I. Perlman, Table of Isotopes, John Wiley and Sons Inc., New York 1967. 